Agréus, L. 248, 256

Aro, P. 256

Bolling-Sternevald, E. 256

Bytzer, P. 237

Carlsson, J. 264

Flook, N. 231

Ford, A.C. 225

Gapasin, J. 218

Halling, K. 264

Holtmann, G. 218

Hongo, M. 231

Jiang, X. 203

Johansson, S.-E. 248

Jones, M.P. 248

Jung, H.K. 203

Junghard, O. 256

Kandulski, A. 210, 243

Kulich, K. 264
Madsen, L.G. 237

Malfertheiner, P. 193, 210, 231, 243

Mimidis, K. 194

Moayyedi, P. 225

Mönkemüller, K. 243

Neumann, H. 243

Ronkainen, J. 256

Ruff, K. 203

Salis, G. 231

Selgrad, M. 210

Sheen, A. 248

Storskrubb, T. 256

Svärdsudd, K. 248

Tack, J. 194

Talley, N.J. 203, 248, 256

Vakil, N. 193, 215, 231

Wiklund, I. 264

\title{
Subject Index Vol. 26, No. 3, 2008
}

Acid related disorders 215

- stimulation 237

Acute gastrointestinal infection 194

Barrett 243

Consultation 248

Dyspepsia 203, 210, 215, 225, 231, 237, $243,248,256,264$

Endoscopy 225

Epidemiology 256

Eradication therapy 210

Erosive reflux disease 243

Evidence based measures 218

Family practice 248

- practitioner 231

Functional dyspepsia 203, 218

- gastrointestinal disorder 203

Gastric accommodation 194

- emptying 194

- sensitivity 194

Gastroesophageal reflux disease 215, 231, 243,256

Gastrointestinal Symptom Rating Scale 264

- symptoms 256

Genetic susceptibility 194
Health care seeking behaviour 248

Health-related quality of life 264

Helicobacter pylori 194, 210, 225

Irritable bowel syndrome 243, 256

Management guidelines 225

Multinational study 264

Non-erosive reflux disease 243

Non-ulcer dyspepsia 203

Omeprazole 237

Patient-reported outcomes 264

Pentagastrin 237

Population study 256

Practice guidelines 231

Primary care physician 231

Proton pump inhibitors 215

Psychosocial factors 194

Quality of life 218

Quality of Life in Reflux and Dyspepsia questionnaire 264

Questionnaire(s) 231, 256

Reflux 231

Response to treatment 237

Survey 231

Symptom severity 264

Treatment 218 